\title{
Mini-Mental State Examination in patients with hepatic encephalopathy and liver cirrhosis: a prospective, quantified electroencephalography study
}

Dorota Koziarska ${ }^{1 \dagger}$, Ewa Wunsch ${ }^{2 *}$, Małgorzata Milkiewicz $^{3}$, Maciej Wójcicki ${ }^{4}$, Przemysław Nowacki ${ }^{1}$ and Piotr Milkiewicz ${ }^{2,4}$

\begin{abstract}
Background: Mini-Mental State Examination (MMSE) is one of the most commonly used methods in the assessment of cognitive mental status. MMSE has been used in hepatology but its usefulness in the evaluation of hepatic encephalopathy (HE) has never been properly assessed. The aim of the study was to investigate the value of MMSE in detection of HE in patients with cirrhosis.

Methods: One hundred and one consecutive patients with liver cirrhosis underwent neurological examination, MMSE and electroencephalography (EEG). Spectral analysis of EEG was done with calculation of mean dominant frequency (MDF) and relative power of delta, theta, alpha and beta rhythms. Minimal HE was diagnosed in patients with normal neurological status and alterations in spectral EEG. Statistical analysis included Fisher's exact and Anova analysis. Categorical data were compared using Levene's test for equality of variances. Correlation-coefficient analysis was performed by the Pearson's $r$ or Z-test, as needed. Tests performance was assessed by the calculating the area under the ROC curve (AUC) and evaluating its difference from reference area ( $A \cup C=0.5$ ). A $p$ value $<0.05$ was considered statistically significant.
\end{abstract}

Results: Overt HE was identified in 49 (48.5\%) and minimal HE in 22 (21.8\%) patients. Although there were significant correlations between both severity of liver disease (Child-Pugh classification), overt HE (West-Haven criteria) and various MMSE items, MDF showed no correlation with any of MMSE items as well as MMSE summary score. MMSE (score and items) did not discriminate patients without HE and minimal HE. The only significant differences between patients without HE and with overt HE were seen in respect of MMSE score $(p<0.02)$, orientation to place $(p<0.003)$, repetition $(p<0.01)$ and complex commands-understanding $(p<0.02)$. Test performance analysis has shown that MMSE has no value as a prediction method in determining minimal HE and in respect of overt HE has a sensitivity of $63 \%$ and specificity of $52 \%$ by a cut-off level at 27.5 points to diagnose overt HE.

Conclusions: In conclusion, although MMSE score and single items are altered in patients with overt HE, MMSE has no value in the assessment of minimal HE. Because MMSE could be impaired in several cognitive dysfunctions, more specific test should be used for measuring HE.

Keywords: Liver cirrhosis, Hepatic encephalopathy, Mini-mental state examination, Electroencephalography, Frequency analysis

\footnotetext{
* Correspondence: ewa.wunsch@gmail.com

${ }^{\dagger}$ Equal contributors

${ }^{2}$ Liver Research Laboratories, Pomeranian Medical University, Al. Powstancow Wlkp. 72, 70-111, Szczecin, Poland

Full list of author information is available at the end of the article
} 


\section{Background}

Hepatic encephalopathy (HE) is a serious complication of liver cirrhosis negatively affecting both health-related quality of life and survival [1-3]. It comprises a plethora of neuropsychiatric symptoms ranging from subtle neurocognitive alterations to severe life-threatening neurological impairment [4]. Although it is now considered as a continuum of neurocognitive deterioration [5], HE is usually divided into minimal and overt HE, regarding the change in the mental state. A characteristic feature of minimal HE is lack of a detectable brain dysfunction in the clinical examination, however subclinical psychomotor slowing and cognition deficits are typically present in minimal $\mathrm{HE}$ and clinically relevant [6-8]. Overt $\mathrm{HE}$ is characterized by various symptoms encompassing neuromuscular abnormalities, intellectual functions, personality and consciousness, traditionally graded on four stages according to West-Haven criteria [3].

Several diagnostic methods were implemented both to diagnose and to monitor $\mathrm{HE}$, but until now there is no consensus about the diagnostic standard. Mini-Mental State Examination (MMSE) has been also used in the assessment of the severity and in monitoring of $\mathrm{HE}$ in several studies [9-13]. MMSE is a brief questionnaire, that was introduced by Folsteinet al. in 1975 for the assessment of cognitive mental status [14]. After minor subsequent modifications it is currently one of the most popular methods for the screening of cognitive function impairment used both in clinical practice and in research in broad variety of medical conditions [15]. MMSE evaluates neurocognitive deficits, that are also present in minimal $\mathrm{HE}$ and are forerunners of more severe HE. These are easily underestimated by a routine examination, therefore the information drew from the detailed analysis of MMSE could potentially serve as a well structured and objective way of identification of changes in mental status in patients with cirrhosis. Nevertheless the real usefullness of MMSE in the evaluation of HE has never been properly studied. Moreover MMSE was until now only interpreted as a total score, and was not divided into separate items. We therefore performed a detailed analysis of MMSE items and investigated the potential value of MMSE in evaluation of $\mathrm{HE}$ in patients with liver cirrhosis.

\section{Methods}

\section{Patients}

One hundred and one consecutive patients with liver cirrhosis: 68 (67.3\%) males and 33 (32.7\%) females, aged $54.5 \pm 11.8$ years (range 18-87) were enrolled to our study. Detailed demographic data are shown in Table 1. Included subjects were both in-patients and out-patients who received their treatment at our institution. Included patients had no history of factors impairing mental status, such as consumption of psychotropic drugs, active alcohol misuse,
Table 1 Demographic data of study group

\begin{tabular}{ll}
\hline Demographic data & Study group (n=101) \\
\hline Age (years, mean \pm SD, range) & $54.5 \pm 11.8(18-87)$ \\
Gender (male/female) & $68(67.3 \%) / 33(32.7 \%)$ \\
Education years (mean \pm SD, range) & $12.0 \pm 2.9(7-19)$ \\
Etiology of cirrhosis & \\
$\quad$ Alcoholic & $38(37.6 \%)$ \\
$\quad$ Autoimmune & $21(20.8 \%)$ \\
$\quad$ Viral hepatitis & $19(18.8 \%)$ \\
$\quad$ Other & $20(19.8 \%)$ \\
$\quad$ Mixed & $3(3.0 \%)$ \\
Previous hepatic encephalopathy & \\
$\quad$ Yes & $34(33.7 \%)$ \\
$\quad$ No & $67(66.3 \%)$ \\
Child-Pugh class & \\
$\quad$ A & $44(43.6 \%)$ \\
B & $35(34.6 \%)$ \\
C & $22(21.8 \%)$ \\
Bilirubin (mg/dl, mean \pm SD, range) & $3.2 \pm 3.6(0.3-21.2)$ \\
INR (mean \pm SD, range) & $1.3 \pm 0.2(1.0-1.8)$ \\
Albumin (mean \pm SD, range) & $3.6 \pm 0.63(2.2-4.9)$ \\
Ammonia (mean \pm SD, range) & $84.2 \pm 98.5(7.1-805)$ \\
\hline
\end{tabular}

neurological (i.e. dementia, epilepsy, alcoholic seizures) and other than liver disease severe medical problems. Liver cirrhosis was diagnosed by liver biopsy and/or a typical appearance of the liver on abdominal ultrasound and/or computed tomography scan. Blood samples for liver biochemistry and venous ammonia level were collected on the day of examination. The Child-Pugh score was calculated to assess the severity of liver disease $[16,17]$. All patients underwent detailed neurological examination, MMSE and closed-eye electroencephalography (EEG) performed by one experienced neurologist (DK).

\section{MMSE}

MMSE consists of several short questions and problems grouped into 8 items: orientation to time and to place, registration, attention and calculation, recall, language skills, repetition and complex commands (i.e. write a sentence of his own choice, reading a sentence, understanding a command, copy of pentagons). Each item is scored and summary score of maximal 30 points is calculated with higher scores indicating better cognitive performance.

\section{EEG analysis}

EEG was recorded for 20 minutes, with eyes closed, in a condition of relaxed wakefulness, using 18 silver-silver chloride electrodes placed according to the International 10-20 system (Nervus-Multimedia EEG, Taugagreininghf, 
Reykjavík, Iceland). The ground and the reference electrode was Fz. The impedance was kept below $5 \mathrm{k} \Omega$. Each channel had its own analog-to-digit converter, the resolution was $0,19 \mathrm{uV} /$ digit. Single observer (DK) visually inspected the traces and classified according to the Parsons- Smith grading [18] modified by Amodio et al. [19].

Spectral analysis was carried out on the transverse derivation $\mathrm{P} 3-\mathrm{Pz}$ and $\mathrm{P} 4-\mathrm{Pz}$ in the frequency range of $0,5-30 \mathrm{~Hz}$. Pre-selected 80-100 s periods of artifact-free recording, split into $5 \mathrm{~s}$ epochs were analyzed by applying Fast Fourier Transformation. Mean dominant frequency (MDF) was calculated, the mean values were used. The relative power of delta $(0.5-4.0 \mathrm{~Hz})$, theta $(4.0-8.0 \mathrm{~Hz})$, alpha $(8.0-13.0 \mathrm{~Hz})$ and beta $(13.0-30.0 \mathrm{~Hz})$ rhythms were calculated. EEG alterations associated with HE were then graded using the thresholds proposed by Amodio et al. [20]. Grade 1 by MDF $>6.8$ and Theta $\% \geq 35 \%$; grade 2 by $\mathrm{MDF} \leq 6.8 \mathrm{~Hz}$ and Delta $\% 49 \%$; grade $3 \mathrm{MDF} \leq 6.8$ and Delta $\% \geq 49$.

\section{Diagnosis of HE}

Clinical evaluation of overt $\mathrm{HE}$ was performed by an experienced neurologist (DK) and comprised of a detailed, comprehensive neurological examination according to West-Haven criteria. Following symptoms were particularly noted: psychomotor slowing, impaired performance of arithmetic, disorientation for time and place, inappropriate behavior, extrapyramidal signs, asterixis, ataxia etc. Patients were also reviewed in respect of neuropsychiatric disorders (e.g. epilepsia, alcoholic seizures) and circadian rhythm disturbance. Overt HE was diagnosed in patients, in whom typical abnormalities were present independent from EEG results. West-Haven criteria were applied for the assessment of the severity of overt $\operatorname{HE}[3,21]$. Minimal HE was considered in patients with unaltered neurological status but with alterations in spectral analysis of EEG (i.e. grade $\geq 1$ according to the system proposed by Amodio P. et al.) [20].

\section{Statistics}

Patient measures are reported as means \pm standard deviation (SD). All data were analyzed using Stat-View-5 Software (SAS Institute, Cary, NC, US) and included Fisher's exact and Anova analysis. Categorical data were compared using Levene's test for equality of variances. Correlation-coefficient analysis was performed by the Pearson's $r$ or Z-test, as needed. Tests performance was assessed by the calculating the area under the ROC curve (AUC) and evaluating its difference from reference area $(A U C=0.5)$. A $p$ value $<0.05$ was considered statistically significant.

\section{Ethics}

Written informed consent was obtained from each patient included in the study. The study protocol was approved by the appropriate ethics committee of Pomeranian Medical University, Szczecin, Poland and conforms to the ethical guidelines of the 1975 Declaration of Helsinki (6th revision, 2008).

\section{Results \\ MMSE results in the study group}

All included patients, who completed MMSE performed three items: registration, language skills and complex command - reading a sentence fully correctly. These items were not further analyzed. MMSE (summary score and most of the items) were independent from age and gender of the patients. Only results for a complex command - copy of pentagons item were more altered in older patients $(p<0.02$, CI95\% $=-0.430--0.053)$. Moreover males had significantly better results in respect of a complex command - write a sentence than females (mean: $0.97 \pm 0.37$ vs $0.84 \pm 0.175$ points, $p<0.03)$.

There were significant correlations between severity of liver disease (Child-Pugh classification) and MMSE summary score $(r=-0.420, p<0.0001$, CI $95 \%=-0.573-0.239)$ and several MMSE items: orientation to time $(r=-0.476$, $p<0.0001$, CI95\% $=-0.618--0.303)$, orientation to place $(r=-0.391, p<0.0001$, CI95\% $=-0.549--0.206)$ and complex commands: understanding a command $(\mathrm{r}=-0.295, p<0.005, \mathrm{CI} 95 \%=-0.468--0.099)$, write $\mathrm{a}$ sentence $(\mathrm{r}=-0.389, p<0.0001, \mathrm{CI} 95 \%=-0.547--0.203)$ and copy of pentagons $(\mathrm{r}=-0.353, p<0.0005, \mathrm{CI} 95 \%=-$ 0.517 - - 0.163). Patients in Child-Pugh class A and B had better results of MMSE (summary score and most of the items) than patients in Child-Pugh class C. In contrast patients in Child-Pugh class A and B achieved similar results in most of the MMSE items. These results are summarized in Table 2.

Venous ammonia concentration did not correlate with MMSE summary score and all items except the repetition item $(\mathrm{r}=-0.324, p<0.002$, CI95\% $=-0.499--0.125)$.

\section{MMSE results in patients with $\mathrm{HE}$}

Neurological examination identified 49 (48.5\%) patients with overt HE: 26 (25.7\%) in grade 1, 16 (15.8\%) in grade 2 and $7(6.9 \%)$ in grade 3 according to West-Haven criteria. In $22(21.8 \%)$ subjects without clinical symptoms of $\mathrm{HE}$, minimal HE was diagnosed based on spectral analysis of EEG.

MMSE (score and items) did not discriminate patients without $\mathrm{HE}$ and minimal $\mathrm{HE}$, however there were significant differences between patients without HE and with overt HE in respect of MMSE summary score $(p<0.02)$, orientation to place $(p<0.003)$, repetition $(p<0.01)$ and complex commands - understanding $(p<0.02)$ (Table 3$)$. In patients with more severe overt HE according to West-Haven criteria we observed reduction in most of MMSE scores, which reached a statistical significance 
Table 2 MMSE results dependent of severity of liver disease (Child-Pugh classification)

\begin{tabular}{|c|c|c|c|c|c|c|}
\hline MMSE categories & Class A $(n=44)$ & Class B $(n=35)$ & Class C ( $n=22)$ & $\mathrm{A} v s \mathrm{~B}$ & A vs C & B vs C \\
\hline MMSE summaryscore & $27.6 \pm 1.9$ & $27.0 \pm 2.0$ & $24.8 \pm 4.8$ & ns & $p<0.0005$ & $p<0.01$ \\
\hline Orientation to time & $5.0 \pm 0.2$ & $4.7 \pm 0.8$ & $4.2 \pm 0.9$ & $p<0.05$ & $p<0.0001$ & $p<0.03$ \\
\hline Orientation to place & $4.9 \pm 0.2$ & $4.8 \pm 0.4$ & $4.4 \pm 0.7$ & ns & $p<0.0001$ & $p<0.001$ \\
\hline Attention and calculation & $3.8 \pm 1.4$ & $3.7 \pm 1.2$ & $3.5 \pm 1.9$ & ns & ns & ns \\
\hline Recall & $2.2 \pm 0.9$ & $2.4 \pm 0.7$ & $1.9 \pm 0.8$ & ns & ns & $p<0.05$ \\
\hline Repetition & $0.8 \pm 0.4$ & $0.8 \pm 0.4$ & $0.7 \pm 0.5$ & ns & ns & ns \\
\hline Complex commands (Understanding) & $2.9 \pm 0.3$ & $2.8 \pm 0.5$ & $2.4 \pm 1.0$ & ns & $p<0.01$ & $p<0.03$ \\
\hline Complex commands (Write a sentence) & $1.0 \pm 0.0$ & $0.9 \pm 0.2$ & $0.8 \pm 0.4$ & ns & $p<0.001$ & $p<0.02$ \\
\hline Complex commands (Copy of pentagons) & $1.1 \pm 0.6$ & $0.9 \pm 0.3$ & $0.6 \pm 0.5$ & ns & $p<0.002$ & ns \\
\hline
\end{tabular}

MMSE Mini-Mental State Examination.

in the following items: orientation to place, attention and a complex command - write a sentence. MMSE summary score was reduced in patients with grade 3 of overt HE in comparison to patients with grade $1 \mathrm{HE}$ (Table 4).

Test performance analysis has shown that MMSE has no value as a prediction method in determining minimal HE (AUC $=0.43, p=0.317$, CI95\%= $0.295-0.561$ ). Although AUC analysis reached the statistical significance in respect of overt $\mathrm{HE}$ (AUC $=0.64, p=0.018$, CI95\% $=0.528-0.752$ ), and by a cut-off level at 27.5 points MMSE summary score had a sensitivity of $63 \%$ and specificity of $52 \%$ to diagnose overt HE.

\section{MDF}

Mean MDF in study group was $7.2 \pm 2.3 \mathrm{~Hz}$ (range 2.3 13.2 Hz). MDF values were independent from subjects' age, however were lower in males than females (mean MDF $6.7 \pm 2.2 \mathrm{~Hz}$ vs $8.2 \pm 2.4 \mathrm{~Hz}$ respectively, $p<0.003$ ) Although MDF correlated significantly with severity of liver disease (i.e. Child-Pugh classification, $\mathrm{r}=-0.373, p=0.0001$, CI95\% $=-0.531--0.191$ ) and HE (i.e. West-Haven classification, $\mathrm{r}=-0.337, p=0.0005$, CI95\% $=-0.5--0.152)$, MDF values showed no correlation with any of MMSE items as good as MMSE summary score (data not shown).

\section{Discussion}

The appropriate diagnosis of HE causes several problems. After exclusion of other causes of mental impairment, a careful clinical examination is usually sufficient for the diagnosis of overt, mainly more advanced stages of $\mathrm{HE}$ (grade 3 and grade 4 i.e. coma). However less advanced overt $\mathrm{HE}$ require a detailed neuropsychological evaluation. Moreover, for detection of minimal HE a clinical examination has a little value because of the lack of clear manifestations, and an additional more sensitive diagnostic tool is in this case necessary. However, until now no method is available that can discriminate the specific stages of $\mathrm{HE}$ objectively through the entire spectrum of minimal and overt HE [4]. The West-Haven grading system, which classifies $\mathrm{HE}$ into categories, remains criticized because of its intense subjectivity and low intra- and inter-observer reliability [5,22]. Therefore several other more objective diagnostic tools (e.g. neuroimaging, neurophysiological methods or psychomotor tests), were implemented to diagnose both minimal and overt HE, but there is still an ongoing debate which of them should be a diagnostic standard [23-25]. In several studies spectral analysis of EEG has been proven to be a valuable, sensitive and observer -independent method to detect minimal HE [19,26],

Table 3 MMSE results in patients without and with HE (minimal and overt)

\begin{tabular}{|c|c|c|c|c|c|c|}
\hline MMSE categories & $\begin{array}{l}\text { Without } \\
\text { HE }(n=30)\end{array}$ & $\begin{array}{l}\text { Minimal } \\
\mathrm{HE}(n=22)\end{array}$ & $\begin{array}{l}\text { Overt HE } \\
(n=49)\end{array}$ & $\begin{array}{l}\text { Without HE } \\
\text { vs minimal HE }\end{array}$ & $\begin{array}{l}\text { Without HE } \\
\text { vs overt } \mathrm{HE}\end{array}$ & $\begin{array}{l}\text { Minimal HE } \\
\text { vs overt HE }\end{array}$ \\
\hline MMSE summaryscore & $27.7 \pm 1.8$ & $27.5 \pm 2.0$ & $25.9 \pm 3.7$ & ns & $p<0.02$ & $p<0.05$ \\
\hline Orientation to time & $4.8 \pm 0.8$ & $4.9 \pm 0.3$ & $4.6 \pm 0.9$ & ns & ns & ns \\
\hline Orientation to place & $4.7 \pm 0.2$ & $4.9 \pm 0.2$ & $4.6 \pm 0.6$ & ns & $p<0.003$ & $p<0.01$ \\
\hline Attention and calculation & $3.7 \pm 1.4$ & $3.8 \pm 1.5$ & $3.6 \pm 1.6$ & ns & ns & ns \\
\hline Recall & $2.4 \pm 0.6$ & $2.1 \pm 0.9$ & $2.1 \pm 0.9$ & ns & ns & ns \\
\hline Repetition & $0.9 \pm 0.3$ & $0.8 \pm 0.4$ & $0.7 \pm 0.5$ & ns & $p<0.01$ & ns \\
\hline Complex commands (Understanding) & $2.9 \pm 0.3$ & $2.9 \pm 0.2$ & $2.6 \pm 0.8$ & ns & $p<0.02$ & $p<0.02$ \\
\hline Complex commands (Write a sentence) & $1.0 \pm 0.2$ & $0.9 \pm 0.2$ & $0.9 \pm 0.3$ & ns & ns & ns \\
\hline Complex commands (Copy of pentagons) & $1.0 \pm 0.2$ & $1.1 \pm 0.9$ & $0.8 \pm 0.4$ & ns & ns & $p<0.01$ \\
\hline
\end{tabular}

MMSE Mini-Mental State Examination, HE hepatic encephalopathy. 
Table 4 MMSE results in patients with overt HE according to West-Haven criteria

\begin{tabular}{|c|c|c|c|c|c|c|}
\hline MMSE categories & Grade $1(n=26)$ & Grade $2(n=16)$ & Grade $3(n=7)$ & Grade 1 vs2 & Grade 1 vs 3 & Grade 2 vs 3 \\
\hline MMSE summary score & $26.8 \pm 2.7$ & $25.4 \pm 3.9$ & $23.7 \pm 5.6$ & ns & $p<0.02$ & ns \\
\hline Orientation to time & $4.7 \pm 0.6$ & $4.4 \pm 0.9$ & $4.4 \pm 1.1$ & ns & ns & ns \\
\hline Orientation to place & $4.8 \pm 0.4$ & $4.4 \pm 0.8$ & $4.4 \pm 0.8$ & $p<0.02$ & $p<0.05$ & ns \\
\hline Attention and calculation & $4.0 \pm 1.2$ & $3.7 \pm 1.6$ & $2.1 \pm 1.9$ & ns & $p<0.003$ & $p<0.02$ \\
\hline Recall & $2.2 \pm 0.8$ & $1.9 \pm 0.9$ & $1.9 \pm 0.9$ & ns & ns & ns \\
\hline Repetition & $0.7 \pm 0.5$ & $0.6 \pm 0.5$ & $0.9 \pm 0.4$ & ns & ns & ns \\
\hline Complex commands (Understanding) & $2.5 \pm 0.7$ & $2.6 \pm 0.9$ & $2.5 \pm 1.1$ & ns & ns & ns \\
\hline Complex commands (Write a sentence) & $1.0 \pm 0.2$ & $0.8 \pm 0.4$ & $0.9 \pm 0.4$ & $p<0.05$ & ns & ns \\
\hline Complex commands (Copy of pentagons) & $0.8 \pm 0.4$ & $0.7 \pm 0.5$ & $0.6 \pm 0.5$ & ns & ns & ns \\
\hline
\end{tabular}

MMSE Mini-Mental State Examination, HE hepatic encephalopathy.

therefore it can be used as a reference tool and we applied it in our study as well. However the EEG detects cortical neuronal activity and may not ideally reflect a wide variety of neurophysiological events seen in HE [24]. Moreover it is not broadly implemented in a daily practice because of its limited availability and the need of an experienced personnel to appropriate interpretation of the obtained electroencephalograms. Therefore there is still a need to develop screening tests for a clinical routine and for a research in studying patients with liver cirrhosis, which will cover a whole spectrum of pathophysiological spectrum of the phenomenon.

Neuropsychological diagnostic tools show a number of practical advantages making them potent candidates for the common use in HE assessment [27,28]. Generally they are simple to perform, not time-consuming can be used in the out-patient clinic. For this purpose both single paper-pencil tests (such as the block design test, the trail making test and the digit symbol test) as well as standardized batteries (i. a. the Repeatable Battery for the Assessment of Neuropsychological Status, the Wechsler Adult Intelligence Scale-Revised, the Auditory Verbal Learning, the Everyday Memory Questionnaire) have been implemented [29-35]. The complex tools have a superior value in comparison to single modalities because they cover a broad spectrum of neurocognitive deficits. However among plenty of these tests only the Psychometric Hepatic Encephalopathy Score (PHES) was especially constructed for detecting minimal HE [36]. Others have a great importance in the diagnosis of cognitive alteration in elderly or neuropsychiatric disorders, in the estimation of the effect of psychoactive drugs or to measure intelligence, but their value in the field of HE remains to be established.

MMSE is one of above mentioned complex neuropsychological tools, that was also occasionally used for identification patients with HE in clinical trials [9-13] and for several reasons could serve as a potential screening test for this purpose. MMSE assesses cognitive abilities: orientation (spatial and time), attention, concentration, calculation, immediate and short-term memory, visuo-spatial orientation, praxis and language skills [14]. Some of these functions are also altered in cirrhotic patients in the course of minimal HE and in stages 1 and 2 overt $\mathrm{HE}$ according to West-Haven criteria, long before neurocognitive decline is obvious and clinically easy to detect [4].

Indeed our study clearly shows that MMSE (summary scores and most of the items) is altered in patients with more advanced liver disease measured by Child-Pugh classification. Likewise we observed an increasing reduction of the most of MMSE items in patients with overt HE. However these differences reached the statistical significance only in few items and mostly in more advanced stages of overt HE (i.e. in grade 2 and 3) and the numeric difference between MMSE scores was small and unlikely to be clinically useful. These included orientation to place, attention and calculation and complex command - writing a sentence of own choice. Moreover the test performance analysis has shown that MMSE summary score has low both specificity and sensitivity in detection of overt HE. Furthermore MMSE summary score and all items did not differentiate patients with minimal HE from those without any signs of HE (i.e. with normal neurological state and without alteration seen in spectral EEG) and did not correlate with MDF values. These observations clearly shows that MMSE should not be considered an appropriate tool for detection of minimal HE and in overt HE in stage 1 and 2 according to West-Haven classification. Although in our study MMSE was altered in patients with stage 3 of overt $\mathrm{HE}$, but this can be detect clinically and generally requires no additional method.

All included into our analysis subjects performed three items: registration, language skills and complex command reading a sentence fully correctly. These observations can be explained by the heterogeneous nature of $\mathrm{HE}$ and by fact that cognitive functions are selectively compromised in this disorder $[37,38]$. While several cognitive functions are altered very early in the natural course of the disorder (i.e. attention, visuo-spatial orientation, psychomotor speed), 
some others are manifestations of more advanced HE (disorientation to place, lethargy, somnolence). On the other hand some intellectual functions such as language skills, long-term memory and intelligence are generally unaffected, even in patients at higher stages of HE $[8,39]$.

\section{Conclusion}

In conclusion, although $\mathrm{HE}$ is a disorder that affects the mental status and causes a decrease in the MMSE, our study strongly suggests that this modality is not an appropriate tool in defining an encephalopathy-related status of patients with cirrhosis and should not be used for this purpose in clinical trials. In view of the complex nature of $\mathrm{HE}$ there is still a need to develop more specific test for objective measuring through the entire spectrum of this phenomenon.

\section{Abbreviations}

MMSE: Mini-Mental State Examination; HE: Hepatic encephalopathy; EEG: Electroencephalography; MDF: Mean dominant frequency.

\section{Competing interests}

The authors declare that they have no competing interests.

\section{Authors' contributions}

DK designed the study, carried out the neurological examination, EEG analysis, performed MMSE and participated in the draft of the manuscript. EW participated in the design of the study, recruited participants and drafted the manuscript. DK and EW contributed equally in the work. MM performed statistical analysis. MW participated in the design and coordination of the study. PN conceived of the study, and participated in its design and coordination. PM participated in the design and coordination of the study and corrected the manuscript. All authors read and approved the final manuscript.

This study was presented at EASL Meeting, Barcelona, Spain 2012, and awarded with the Young Investigator Bursary.

\section{Author details}

${ }^{1}$ Department of Neurology, Pomeranian Medical University, ul. Unii Lubelskiej 1, 71-252, Szczecin, Poland. ' Liver Research Laboratories, Pomeranian Medical University, Al. Powstancow Wlkp. 72, 70-111, Szczecin, Poland. ${ }^{3}$ Medical Biology Laboratory, Pomeranian Medical University, Al. Powstancow Wlkp. 72, 70-111, Szczecin, Poland. ${ }^{4}$ Department of General, Transplant and Liver Surgery of the Medical University of Warsaw, Warsaw, Poland.

Received: 15 December 2012 Accepted: 28 June 2013

Published: 2 July 2013

\section{References}

1. Arguedas MR, DeLawrence TG, McGuire BM: Influence of hepatic encephalopathy on health-related quality of life in patients with cirrhosis. Dig Dis Sci 2003, 48:1622-1626.

2. Bustamante J, Rimola A, Ventura PJ, Navasa M, Cirera I, Reggiardo V, Rodes J: Prognostic significance of hepatic encephalopathy in patients with cirrhosis. J Hepatol 1999, 30:890-895.

3. Ferenci P, Lockwood A, Mullen K, Tarter R, Weissenborn K, Blei AT: Hepatic encephalopathy--definition, nomenclature, diagnosis, and quantification: final report of the working party at the 11th World Congresses of Gastroenterology, Vienna, 1998. Patology 2002, 35:716-721.

4. Bajaj JS, Wade JB, Sanyal AJ: Spectrum of neurocognitive impairment in cirrhosis: implications for the assessment of hepatic encephalopathy. Patology 2009, 50:2014-2021.

5. Bajaj JS, Cordoba J, Mullen KD, Amodio P, Shawcross DL, Butterworth RF, Morgan MY: Review article: the design of clinical trials in hepatic encephalopathy-an International Society for Hepatic Encephalopathy and Nitrogen Metabolism (ISHEN) consensus statement. Aliment Pharmacol Ther 2011, 33:739-747.

6. Wein C, Koch H, Popp B, Oehler G, Schauder P: Minimal hepatic encephalopathy impairs fitness to drive. Patology 2004, 39:739-745.

7. Romero-Gomez M, Boza F, Garcia-Valdecasas MS, Garcia E, Aguilar-Reina J: Subclinical hepatic encephalopathy predicts the development of overt hepatic encephalopathy. Am J Gastroenterol 2001, 96:2718-2723.

8. Amodio P, Montagnese S, Gatta A, Morgan MY: Characteristics of minimal hepatic encephalopathy. Metab Brain Dis 2004, 19:253-267.

9. Jover R, Company L, Gutierrez A, Zapater P, Perez-Serra J, Girona E, Aparicio $J R$, Perez-Mateo M: Minimal hepatic encephalopathy and extrapyramidal signs in patients with cirrhosis. Am J Gastroenterol 2003, 98:1599-1604.

10. Kramer L, Bauer E, Gendo A, Funk G, Madl C, Pidlich J, Gangl A: Neurophysiological evidence of cognitive impairment in patients without hepatic encephalopathy after transjugular intrahepatic portosystemic shunts. Am J Gastroenterol 2002, 97:162-166.

11. Malaguarnera M, Gargante MP, Cristaldi E, Vacante M, Risino C, Cammalleri L, Pennisi G, Rampello L: Acetyl-L-carnitine treatment in minimal hepatic encephalopathy. Dig Dis Sci 2008, 53:3018-3025.

12. Meyer T, Eshelman A, Abouljoud M: Neuropsychological changes in a large sample of liver transplant candidates. Transplant Proc 2006, 38:3559-3560.

13. Yoo HY, Edwin D, Thuluvath PJ: Relationship of the model for end-stage liver disease (MELD) scale to hepatic encephalopathy, as defined by electroencephalography and neuropsychometric testing, and ascites. Am J Gastroenterol 2003, 98:1395-1399.

14. Folstein MF, Folstein SE, McHugh PR: "Mini-mental state". A practical method for grading the cognitive state of patients for the clinician. J Psychiatr Res 1975, 12:189-198.

15. Crum RM, Anthony JC, Bassett SS, Folstein MF: Population-based norms for the Mini-Mental State Examination by age and educational level. JAMA 1993, 269:2386-2391.

16. Child CG, Turcotte JG: Surgery and portal hypertension. Jor Prob/ Clin Surg 1964, 1:1-85.

17. Pugh RN, Murray-Lyon IM, Dawson JL, Pietroni MC, Williams R: Transection of the oesophagus for bleeding oesophageal varices. J Surg 1973, 60:646-649.

18. Summerskill WH, Parsons-Smith BG, Dawson AM, Sherlock S: The electroencephalograph in liver disease. Lancet 1957, 273:867-871.

19. Amodio P, Del PF, Petteno E, Mapelli D, Angeli P, lemmolo R, Muraca M, Musto C, Gerunda G, Rizzo C, Merkel C, Gatta A: Prevalence and prognostic value of quantified electroencephalogram (EEG) alterations in cirrhotic patients. J Hepatol 2001, 35:37-45.

20. Amodio P, Campagna F, Olianas S, lannizzi P, Mapelli D, Penzo M, Angeli P, Gatta A: Detection of minimal hepatic encephalopathy: normalization and optimization of the Psychometric Hepatic Encephalopathy Score. A neuropsychological and quantified EEG study. J Hepatol 2008, 49:346-353.

21. Atterbury CE, Maddrey WC, Conn HO: Neomycin-sorbitol and lactulose in the treatment of acute portal-systemic encephalopathy. A controlled, double-blind clinical trial. Am J Dig Dis 1978, 23:398-406.

22. Kircheis G, Fleig WE, Gortelmeyer R, Grafe S, Haussinger D: Assessment of low-grade hepatic encephalopathy: a critical analysis. J Hepatol 2007, 47:642-650

23. Bajaj JS: Current and future diagnosis of hepatic encephalopathy. Metab Brain Dis 2010, 25:107-110.

24. Guerit JM, Amantini A, Fischer C, Kaplan PW, Mecarelli O, Schnitzler A, Ubiali E, Amodio P: Neurophysiological investigations of hepatic encephalopathy: ISHEN practice guidelines. Liver Int 2009, 29:789-796.

25. Montagnese S, Amodio P, Morgan MY: Methods for diagnosing hepatic encephalopathy in patients with cirrhosis: a multidimensional approach. Metab Brain Dis 2004, 19:281-312.

26. Amodio P, Pellegrini A, Ubiali E, Mathy I, Piccolo FD, Orsato R, Gatta A, Guerit JM: The EEG assessment of low-grade hepatic encephalopathy: comparison of an artificial neural network-expert system (ANNES) based evaluation with visual EEG readings and EEG spectral analysis. Clin Neurophysiol 2006, 117:2243-2251.

27. Randolph C, Hilsabeck R, Kato A, Kharbanda P, Li YY, Mapelli D, Ravdin LD, Romero-Gomez M, Stracciari A, Weissenborn K: Neuropsychological assessment of hepatic encephalopathy: ISHEN practice guidelines. Liver Int 2009, 29:629-635.

28. Montagnese S, Schiff S, De RM, Crossey MM, Amodio P, Taylor-Robinson SD: Neuropsychological tools in hepatology: a survival guide for the clinician. J Viral Hepat 2012, 19:307-315. 
29. Loguercio C, Del Vecchio-Blanco C, Coltorti M: Psychometric tests and "latent" portal-systemic encephalopathy. Br J Clin Pract 1984, 38:407-411.

30. Malaguarnera M, Greco F, Barone G, Gargante MP, Malaguarnera M, Toscano MA: Bifidobacterium longum with fructo-oligosaccharide (FOS) treatment in minimal hepatic encephalopathy: a randomized, double-blind, placebo-controlled study. Dig Dis Sci 2007, 52:3259-3265.

31. Malaguarnera M, Vacante M, Motta M, Giordano M, Malaguarnera G, Bella R, Nunnari G, Rampello L, Pennisi G: Acetyl-L-carnitine improves cognitive functions in severe hepatic encephalopathy: a randomized and controlled clinical trial. Metab Brain Dis 2011, 26:281-289.

32. Montagnese S, Schiff S, Turco M, Bonato CA, Ridola L, Gatta A, Nousbaum $J$ B, Riggio O, Merkel C, Amodio P: Simple tools for complex syndromes: a three-level difficulty test for hepatic encephalopathy. Dig Liver Dis 2012, 44:957-960

33. Randolph C, Tierney MC, Mohr E, Chase TN: The repeatable battery for the assessment of neuropsychological status (RBANS): preliminary clinical validity. J Clin Exp Neuropsychol 1998, 20:310-319.

34. Sood GK, Sarin SK, Mahaptra J, Broor SL: Comparative efficacy of psychometric tests in detection of subclinical hepatic encephalopathy in nonalcoholic cirrhotics: search for a rational approach. Am J Gastroenterol 1989, 84:156-159.

35. Weissenborn $\mathrm{K}$, Ruckert N, Hecker H, Manns MP: The number connection tests $A$ and $B$ : interindividual variability and use for the assessment of early hepatic encephalopathy. J Hepatol 1998, 28:646-653.

36. Weissenborn K, Ennen JC, Schomerus H, Ruckert N, Hecker H: Neuropsychological characterization of hepatic encephalopathy. J Hepatol 2001, 34:768-773.

37. Haussinger D, Schliess F: Pathogenetic mechanisms of hepatic encephalopathy. Gut 2008, 57:1156-1165.

38. Blei AT, Cordoba J: Hepatic encephalopathy. Am J Gastroenterol 2001, 96:1968-1976.

39. Weissenborn K, Giewekemeyer K, Heidenreich S, Bokemeyer M, Berding G, Ahl B: Attention, memory, and cognitive function in hepatic encephalopathy. Metab Brain Dis 2005, 20:359-367.

doi:10.1186/1471-230X-13-107

Cite this article as: Koziarska et al:: Mini-Mental State Examination in patients with hepatic encephalopathy and liver cirrhosis: a prospective, quantified electroencephalography study. BMC Gastroenterology 2013 13:107.

\section{Submit your next manuscript to BioMed Central and take full advantage of:}

- Convenient online submission

- Thorough peer review

- No space constraints or color figure charges

- Immediate publication on acceptance

- Inclusion in PubMed, CAS, Scopus and Google Scholar

- Research which is freely available for redistribution 